\title{
Effectiveness of psychological interventions to improve quality of life in people with long-term conditions: rapid systematic review of randomised controlled trials
}

\author{
Niall Anderson ${ }^{1,2^{*}}$ and Gozde Ozakinci
}

\begin{abstract}
Background: Long-term conditions may negatively impact multiple aspects of quality of life including physical functioning and mental wellbeing. The rapid systematic review aimed to examine the effectiveness of psychological interventions to improve quality of life in people with long-term conditions to inform future healthcare provision and research.

Methods: EBSCOhost and OVID were used to search four databases (PsychInfo, PBSC, Medline and Embase). Relevant papers were systematically extracted by one researcher using the predefined inclusion/exclusion criteria based on titles, abstracts, and full texts. Randomized controlled trial psychological interventions conducted between 2006 and February 2016 to directly target and assess people with long-term conditions in order to improve quality of life were included. Interventions without long-term condition populations, psychological intervention and/or patient-assessed quality of life were excluded.

Results: From 2223 citations identified, 6 satisfied the inclusion/exclusion criteria. All 6 studies significantly improved at least one quality of life outcome immediately post-intervention. Significant quality of life improvements were maintained at 12-months follow-up in one out of two studies for each of the short- (0-3 months), medium- (3-12 months), and longterm ( $\geq 12$ months) study duration categories.

Conclusions: All 6 psychological intervention studies significantly improved at least one quality of life outcome immediately post-intervention, with three out of six studies maintaining effects up to 12-months post-intervention. Future studies should seek to assess the efficacy of tailored psychological interventions using different formats, durations and facilitators to supplement healthcare provision and practice.
\end{abstract}

Keywords: Long-term, Physical, Conditions, Psychological, Intervention, Health, Quality, Life, Mental, Wellbeing

\section{Background}

Long-term conditions (LTC) are complex physical health issues that last a year or longer and require ongoing care and support [1]. As LTC may be treated but not reversed, long-term care for patients and specialised rehabilitation training for staff is required to deal with the permanent and/or disabling nature of conditions $[1,2]$. As a conse-

\footnotetext{
* Correspondence: nca2@st-andrews.ac.uk

'Public Health Department, NHS Borders, Melrose TD6 9BD, UK

${ }^{2}$ School of Medicine, University of St Andrews, St Andrews KY16 9TF, UK
}

quence of increased exposure to risk factors, the likelihood of experiencing a LTC shows a linear increase with age, with those aged 75 years or older being up to five times more likely to experience a LTC than any other age group $[1,3,4]$. As the proportion of those aged 65 years or older in Europe is projected to increase from 15\% in 2000 to $23.5 \%$ in 2030, a major and increasing challenge is faced by public health to not only target LTC symptoms, but also the associated increased rates of disability and reductions in both healthy and overall life expectancy $[5,6]$. Furthermore, due to LTC resulting from a combination of genetic, physiological, psychological and socio-economic factors, 
LTC are also becoming increasingly prevalent in younger populations [6].

LTC encompass a wide range of conditions which impact upon one's physical, psychological, and social functioning. However, as individual LTC may differ in aetiology, presentation and consequence, there is significant variability in the degree to which each LTC is medically understood, diagnosed and treated [1, 6, 7]. For example, cardiovascular disease and diabetes mellitus are two of the most prevalent and increasingly occurring LTC worldwide, and are associated with increased rates of long-term disability, dependency on others for everyday functioning, and depression [6, 8-10]. Chronic obstructive pulmonary disease and dementia are prevalent but under-diagnosed LTC as symptoms may often be mistakenly attributed to an anticipated gradual age-related decline in functioning. However, both conditions relate to increased medical admissions, distressing symptoms, mortality, and disability [6, 11-13]. Medically unexplained physical symptoms (MUPS) - such as chronic fatigue syndrome, irritable bowel syndrome and fibromyalgia - are also LTC that (despite having unknown aetiologies) profoundly impact psychological, emotional and physical functioning, as well as healthcare costs and requirements [14-16]. Furthermore, aforementioned conditions only provide a snapshot of overall LTC types, and disorder-related fatalities are also predicted to increase for manageable conditions such as asthma without further public health intervention [6].

While it is important to understand the causes, presentations, and consequences of LTC in isolation, to effectively understand the burden of LTC it is critical to look at how multiple LTC may co-occur and interact. While the terms 'Multi-morbidity' and 'Co-morbidity' are often used interchangeably, the former refers to several LTC coexisting, while the latter refers to multiple disorders stemming from one predominant LTC $[17,18]$. Effective determination of the worldwide rates of specific and multi-morbid LTC is complex because of issues with insufficient or inappropriate health measures and analyses being used, and between-country differences in LTC definitions and inclusion criteria [19, 20]. However, regardless of the figures assessed, LTC pose a key challenge as $14-29 \%$ of the European population report one LTC and $7-18 \%$ report two or more conditions [21]. Furthermore, these conservative estimates consider a limited range of conditions, and when a broader range of LTC is considered these figures may be considerably higher. For example, 27\% of 75-84 year olds in Scotland experience two or more LTC [1]. Hence, policy and interventions must not only target specific LTC, but also account for the often multi-morbid nature of LTC.

Health status is an effective measure of healthcare and intervention effectiveness; however, using solely population- level mortality and morbidity rates may be problematic as they only provide a snapshot of effects [22]. As a consequence, subjective measures such as quality of life (QOL), health-related QOL (HR-QOL) and mental wellbeing (MWB) are increasingly being used in healthcare research to assess subjective health status and condition-related burden and coping [22]. QOL is a multi-dimensional concept that includes subjective evaluations of one's physical, psychological, emotional, social, functional and/or environmental state. Due to the wide range of potential constructs, QOL may be assessed using uni-dimensional, multidimensional, and individual measures [23-33]. HR-QOL and MWB are sub-domains of QOL that may be assessed using general or specific measures [23, 34-43]. HR-QOL relates to one's perception of physical and mental health and may provide a valuable insight into symptomology-psychology links, while MWB relates to one's ability to cope with life stressors and maintain a healthy mental state which may provide an insight into illness and coping perceptions [23, 34-43].

LTC diagnosis, treatment, and outcomes not only have a significant impact upon patients' physical functioning, but may also have profound consequences for psychological wellbeing and QOL through affecting emotional, physiological and MWB. This may consequently impact upon medical outcomes through treatment choice and the likelihood of LTC relapse and survival [44-50]. Comorbid mental health disorders are a key issue in LTC populations [11], with LTC patients being significantly more likely to be diagnosed with depressive and/or anxiety disorders $[51,52]$. This may relate to poorer health outcomes and self-care, more severe symptoms, reduced medical adherence, and increased unhealthy behaviours, healthcare spending, and disorder-related death rates [51, 52]. Despite this, traditional medical models often overlook key psychological variables through employing a paternalistic care approach where clinicians exercise predominant authority over patients' care [53-55]. Therefore, as LTC outcomes not only relate to healthcare treatment but are also intrinsically linked to psychological wellbeing and mental health, the provision of psychological interventions and therapies is critical for LTC healthcare services and patient outcomes $[11,56,57]$.

Previous systematic reviews (SR) have demonstrated efficacy for psychological interventions (provided in a wide range of formats) to improve both QOL and physical health outcomes in specific LTC patients. For example, mindfulness for multiple sclerosis and cancer, psychosocial interventions for diabetes and cancer, cognitive behavioural therapy (CBT) and relaxation for recurrent headaches, and internet-based CBT or coaching for chronic somatic conditions [58-66]. However, to the researchers' knowledge, there has not previously been a SR that attempts to only assess studies with high scientific rigour 
that utilise psychological interventions across LTC in order to provide valid comparisons for the effectiveness of interventions and guide LTC healthcare development. As aforementioned, as research has demonstrated that LTC may have profound physiological and psychological effects $[1,6,8-16]$, rates of specific and multi-morbid LTC are high and predicted to rise $[3-6,17,18,21]$, and psychological interventions may improve both QOL and physical functioning [56-66], it is crucial to determine which interventions may be effective across conditions.

The rapid SR aimed to examine the effectiveness of a variety of psychological interventions that seek to improve generic or specific QOL, HR-QOL and/or MWB in people with LTC to determine whether specific interventions may be viable and efficacious for general LTC healthcare implementation. As randomised controlled trial (RCT) designs are the most rigorous and effective method for determining whether intervention-outcome relationships are present [67], and to ensure valid comparisons were possible between studies, only RCTs with a usual care control (UCC) condition which directly target and assess patients with a current LTC diagnosis were included. To ensure the review assessed the most up-to-date research, only studies published between 2006 and February 2016 were included. Furthermore, despite a general dose and duration effect being present for psychological intervention effectiveness, evidence relating to the optimum duration of psychological interventions for LTC to achieve maximum effectiveness is mixed [62, 68, 69]. Therefore, an ante hoc decision was taken to categorise studies by intervention facilitation duration, encompassing short(0-3 months), medium- (3-12 months) and long-term ( $\geq 12$ months) study classifications.

\section{Methods}

\section{Rapid systematic review}

Rapid SR are a form of streamlined SR that may be used by healthcare professionals to guide policy in a timeframe that may not be possible using traditional SR methods. While they do not provide as in-depth information and should not be viewed as a substitute for traditional SRs, rapid SR may have important implications for healthcare decision-making through using systematic methods to provide high-quality information and draw significantly similar conclusions to a traditional SR [70-72]. As the review was conducted during NHS employment and aimed to influence healthcare policy, utilizing a SR procedure was deemed the most feasible and practical approach based on two key considerations. First, in order for the research to have implications (not only for research but also) for healthcare, it was critical that high quality information was provided using limited time and resources [70]. Second, as the research was conducted during NA's NHS employment as one competency of a two-year professional doctoratelevel Health Psychology qualification, the ability to generate a complete draft of findings for NHS stakeholders within a maximum of 6 months (as opposed to up to 2 years for a traditional SR) [70-72] was deemed the most appropriate approach. Therefore, two researchers (NA, GO) followed traditional SR procedures but without searching grey literature and with only one researcher (NA) involved until data extraction was completed. The implications of adopting this approach are presented in 'Rapid Systematic Review Strengths and Limitations'.

\section{Search strategy, selection criteria and data extraction}

Searches were conducted on 19.02.2016 by one researcher (NA) using EBSCOhost to access PsychInfo (1967-2016) and PBSC (1974-2016), and OVID to access Medline (1946-2016) and Embase (1974-2016). Both databases were searched using key terms (Table 1), with potential citations suitability assessed using the predefined inclusion/exclusion criteria (Table 2). Due to the multi-dimensional nature of QOL there is currently no universally accepted definition of QOL $[22,25]$. Therefore, an ante hoc decision was made to manually assess individual studies for the presence or absence of QOL rather than include it in the search terms. Additionally, only RCTs with a UCC were included in order to ensure that valid comparisons of rigour and effectiveness were possible between different interventions and LTC [67]. Data were extracted using a template developed from the COCHRANE criteria [73]. As the SR aimed to guide public health policy, the Effective Public Health Practice Project (EPHPP) 'Quality Assessment Tool for Quantitative Studies' was used to assess study quality [74].

\section{Results}

Study selection

The PRISMA flowchart (Fig. 1) demonstrates the process used to narrow 2224 prospective citations to 13 studies based on titles and abstracts [75-87], with 6 studies satisfying the inclusion/exclusion criteria based on full articles [82-87].

\section{Study characteristics}

Key study features, measures, results (including significance values and effect sizes where stated), and authors' conclusions from the 6 eligible studies are presented in Table 3. The six studies [82-87] encompass a variety of psychological interventions and durations: 2 were shortterm (0-3 months) [82, 85], 2 were medium-term (312 months) [84, 86], and 2 were long-term studies ( $\geq 12$ months) [83, 87]. Facilitators of the interventions varied considerably between studies, with nurses facilitating 3 interventions [83, 85, 87], and the remaining 3 studies being facilitated by health educators [82], CBT 
Table 1 Database Search Terms

\begin{tabular}{lllll}
\hline Stage & Criteria & EBSCO & OVID & Combined \\
\hline 1 & (psych* AND interven*) $^{*}$ & 242,630 & 333,035 & 575,665 \\
2 & AND ((long* AND term* AND & 793 & 1430 & 2223 \\
& $\begin{array}{l}\text { physical* AND condition*) } \\
\text { OR ((persist* AND physical* }\end{array}$ & & & \\
& AND health) AND (issue* & & & \\
& OR problem*))) & & \\
\hline
\end{tabular}

therapists [84], and clinical psychologists [86]. Additionally, each intervention focussed on a different LTC; comprising asthma [82], human immunodeficiency virus (HIV) [83], MUPS [84], congestive heart failure (CHF) [85], knee osteoarthritis [86], and head \& neck cancer (HNC) patients [87]. Five studies compared a UCC with one intervention [82-85, 87], while one study contrasted multiple interventions with a UCC [86]. Furthermore, all 6 studies comprised samples of both genders aged 18 years or over, and assessed (among other measures) generic and/or specific measures of QOL, HR-QOL and/ or MWB [82-87].

\section{Study quality assessment}

EPHPP quality assessment [74] involves assessing studies based on 6 key components (Table 4). Each component comprises multiple choice questions for which scores are combined to provide an overall component rating of 'Strong,' 'Moderate' or 'Weak'. All component ratings are then combined to provide an overall quality rating of 'Strong' for no 'Weak' components, 'Moderate' for one 'Weak' component, and 'Weak' for two or more 'Weak' components.

\section{Short-term interventions (0-3 months)}

Two short-term interventions were present. Baptist et al. [82] offered a 6-week health educator-led self-regulation intervention for asthmatic patients $(N=70)$, comprising 3 consecutive weekly health education group sessions followed by 3 weekly one-to-one telephone sessions. Health educators received a 2-day training session on selfregulation and asthma management principles which was used to conduct tailored self-regulation interventions. This involved patients' self-selecting a specific asthmarelated problem that they wished to address before planning how to achieve positive outcomes and cope with potential asthma-related issues. Significant improvements were present 12-months post-intervention for overall asthma-related QOL, activity, control and hospitalisations. QOL symptom and environment improvements were present 1-month post-intervention, and non-significant changes occurred for QOL emotions or emergency department usage.

Table 2 Review Selection Criteria

\begin{tabular}{|c|c|c|}
\hline Component & Inclusion & Exclusion \\
\hline \multirow[t]{5}{*}{ Population } & $\begin{array}{l}\text { Any LTC (including MUPS) not limited to the conditions } \\
\text { discussed in the introduction e.g. kidney or inflammatory } \\
\text { bowel disease }\end{array}$ & Mental health or psychiatric conditions in the absence of LTC \\
\hline & $\begin{array}{l}\text { Any age group from school-aged adolescents ( } \geq 10 \text { years) } \\
\text { onwards in order to ensure appropriate levels of } \\
\text { understanding and communication of QOL domains }\end{array}$ & Pre-school or primary school children (0-9 years) \\
\hline & Any gender & No gender exclusions \\
\hline & Any cultural, education or socio-economic status & No cultural, education or socio-economic exclusions \\
\hline & Any care setting or delivery format & No care setting or delivery format exclusions \\
\hline \multirow[t]{3}{*}{ Intervention } & $\begin{array}{l}\text { Psychological intervention (in any format) including those } \\
\text { which include alternative but related terminology e.g. } \\
\text { cognitive behavioural therapy (CBT) or mindfulness }\end{array}$ & Non-psychological interventions \\
\hline & Target and assess LTC patients directly & $\begin{array}{l}\text { Psychological interventions designed to indirectly target LTC } \\
\text { patients (through clinicians, family, carers etc.) }\end{array}$ \\
\hline & Any facilitator & No facilitator exclusions \\
\hline \multirow[t]{4}{*}{ Study Design } & RCT (Level I Quantitative evidence) & $\begin{array}{l}\text { Levels II-V Quantitative evidence, qualitative studies, book chapters, } \\
\text { dissertations, SR and meta-analysis papers, unpublished journals or } \\
\text { grey material }\end{array}$ \\
\hline & Journal articles published in English & Non-English publications \\
\hline & $\begin{array}{l}\text { Comparisons made between intervention and UCC at all } \\
\text { relevant points }\end{array}$ & No intervention and/or UCC conditions \\
\hline & Published between 2006 and February 2016 & Published prior to 2006 and after February 2016 \\
\hline \multirow[t]{2}{*}{ Outcomes } & $\mathrm{QOL}, \mathrm{HR}-\mathrm{QOL}$ and/or WMB & Non-psychological assessment \\
\hline & Assess patients directly & $\begin{array}{l}\text { Measures that indirectly assess patients (through clinicians, family, } \\
\text { carers etc.) }\end{array}$ \\
\hline
\end{tabular}




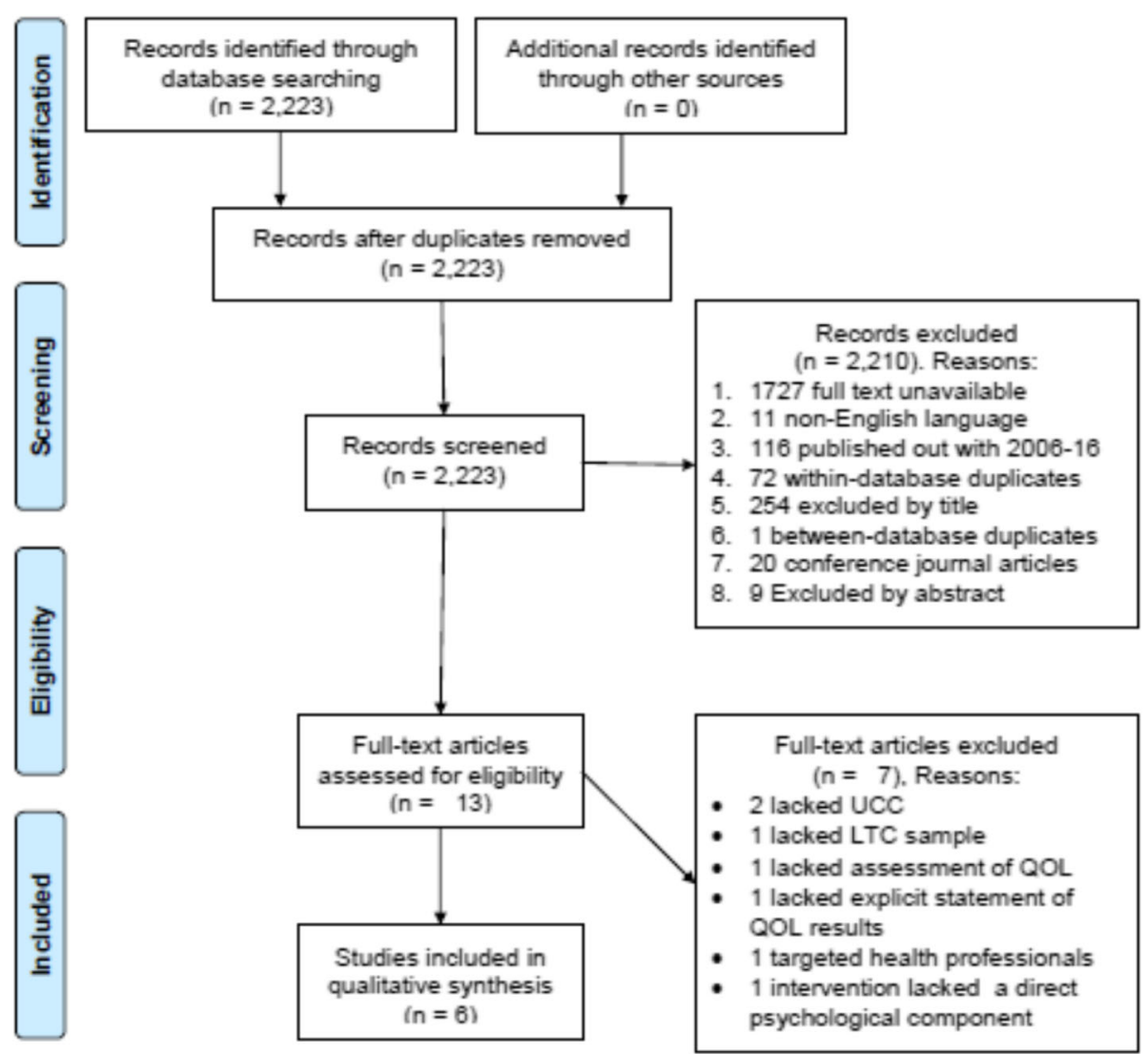

Fig. 1 Study Selection Process

Smeulders et al. [85] offered a 6-week, 150-min per week structured self-management programme for $\mathrm{CHF}$ patients $(n=317)$. The intervention was co-facilitated by a cardiac nurse specialist and a CHF patient (acting as a peer role model) who were both trained on a 4-day 'Chronic Disease Self-Management Programme' [88] by a research and CHF nurse specialist. This incorporated four strategies to enhance self-efficacy over one's condition: skills mastery, behaviour modelling, social persuasion and symptom reinterpretation. Significant improvements were present immediately (but not at 6 - or 12 -months) postintervention for cardiac-specific QOL, cognitive symptom management and self-care behaviour. However, nonsignificant intervention effects were present at all timepoints for perceived control, general self-efficacy, and all other QOL outcomes (general QOL, perceived autonomy, and anxiety and depression).

\section{Medium-term interventions (3-12 months)}

Two medium-term interventions were present. Escobar et al. [84] offered 10, 45-60-min CBT therapist-led sessions over a 3 -month period to MUPS patients $(n=172)$. Two therapists received training from two authors employed by Departments of Psychology and Psychiatry respectively, with protocol adherence routinely evaluated using "taped" recordings. Key topics included managing physical distress, relaxation, activity regulation, emotional awareness, cognitive restructuring and interpersonal communication. The intervention significantly improved patient-rated depression and current somatic symptoms, and physician-rated global severity of symptoms, immediately post-intervention. Only changes to patient-rated somatic symptoms were maintained 6months post-intervention and no effects were present for anxiety or physical functioning.

Somers et al. [86] 'Pain Coping Skills Training' (PCST) and 'Behavioural Weight Management' (BWM) cointerventions for knee osteoarthritis patients $(n=232)$ were conducted by clinical psychologists (with 1-6 years experience in their respective area), under the supervision and training of an experienced senior clinical psychologist. The intervention spanned 24 weeks, comprising 12 weekly groups sessions followed by 12 weeks of sessions every second week for the remainder of the intervention. One group received BWM based on the 'LEARN' programme [89], which focused on lifestyle, exercise, attitudes, relationships and nutrition. The second group received PCST, which focused on maladaptive pain catastrophizing and 


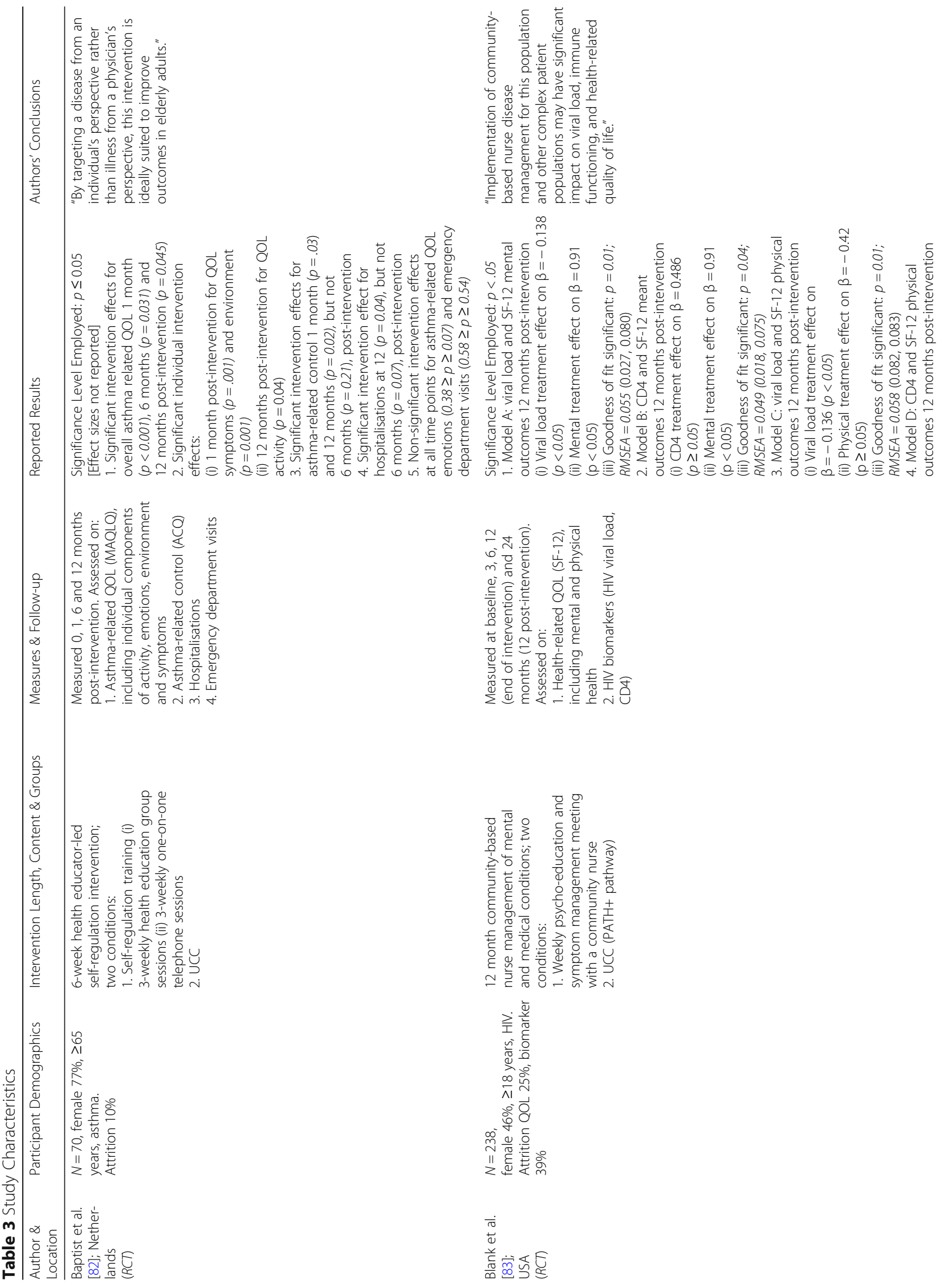




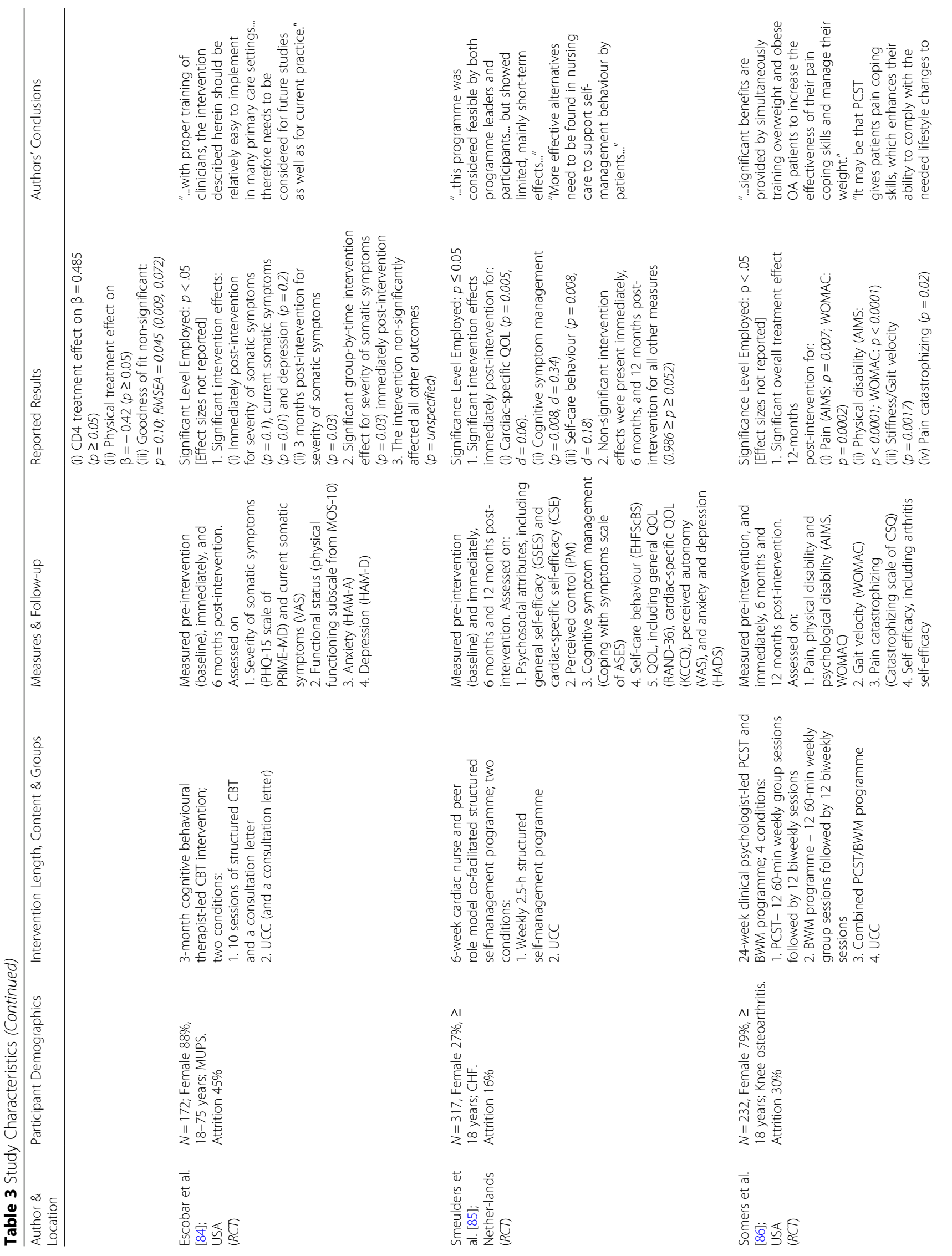




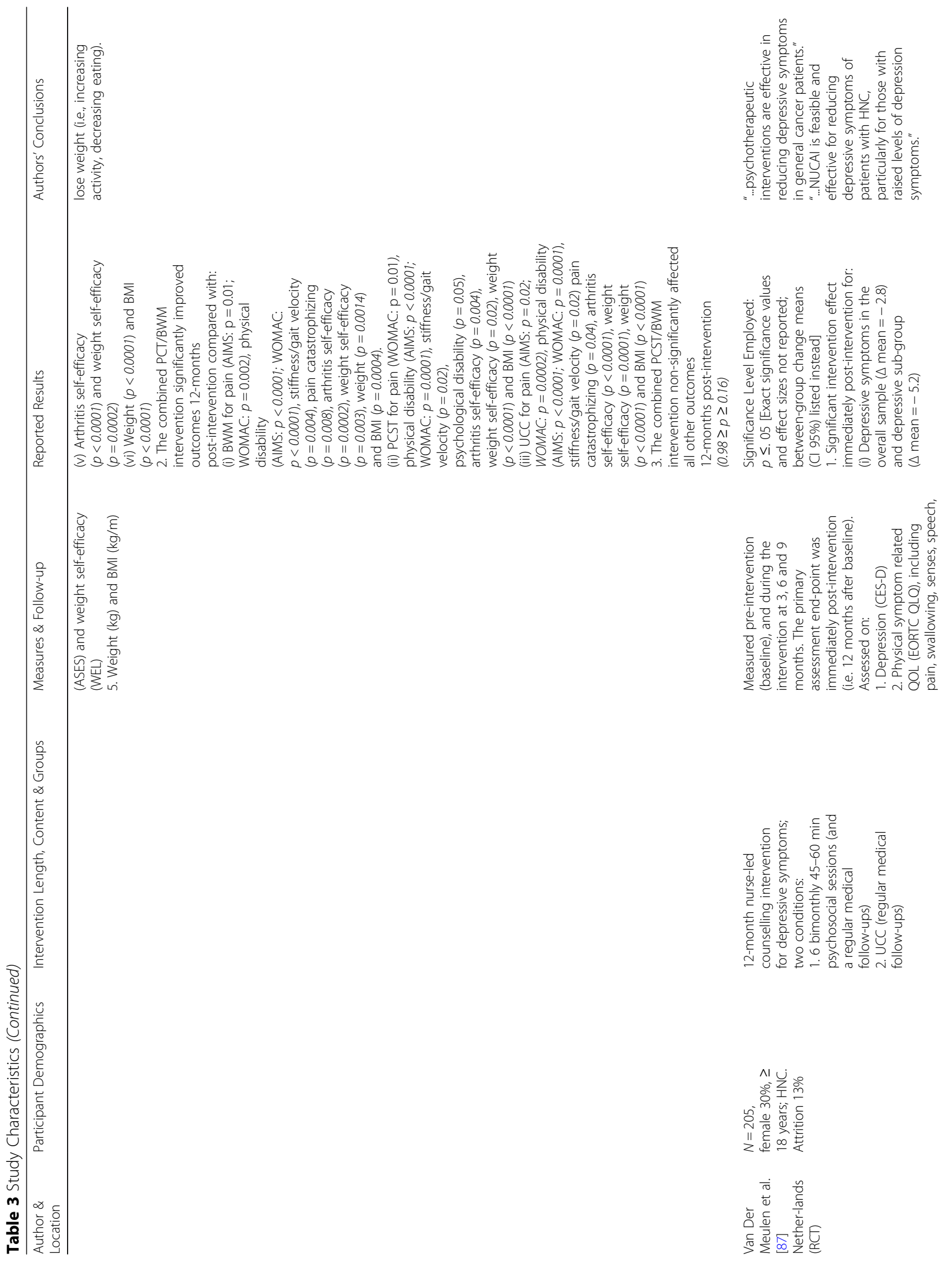




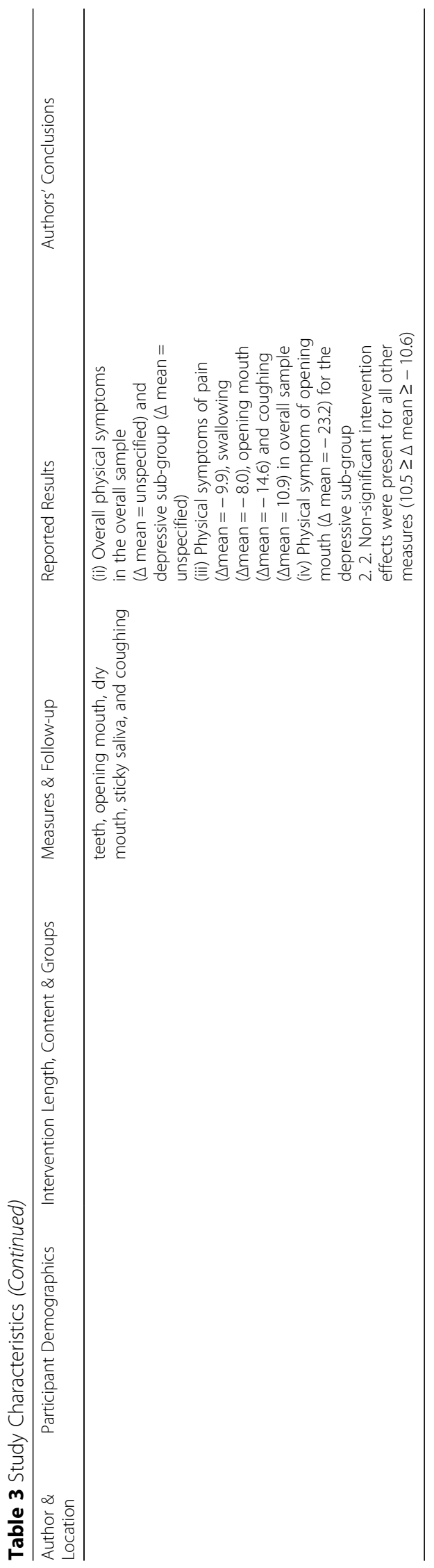


Table 4 EPHPP Quality Assessment

\begin{tabular}{llllllll}
\hline Study & \multicolumn{2}{l}{ EPHPP Sub-domains } & & & EPHPP \\
\cline { 2 - 7 } & Selection Bias & Design & Confounders & Blinding & Data Collection & Withdrawals \& Dropouts & Overall Rating \\
\hline Baptist [82] & W & S & S & S & S & S & M \\
Blank [83] & S & S & S & S & S & W & M \\
Escobar [84] & S & S & S & M & S & S & W \\
Smeulders [85] & W & S & S & W & S & M & S \\
Somers [86] & S & S & S & M & S & S & S \\
Van Der Meulen [87] & M & S & S & S & S & &
\end{tabular}

Study Quality Rating: W: Weak; M: Moderate; S: Strong

adaptive coping strategies. The third group received both BWM and PCST programmes. While the study did not utilise a generic measure of QOL, the combined intervention demonstrated significant improvements compared to UCC 12-months post-intervention for arthritis- and weight-specific self-efficacy, pain symptoms and catastrophizing, physical disability and stiffness, weight, and BMI.

\section{Long-term interventions ( $\geq 12$ months)}

Two long-term interventions were present. Blank et al. [83] offered weekly community-based psycho-education and symptom management sessions (of unspecified duration) over a 12 -month period to HIV patients $(n=238)$. Four Advanced Practice Nurses facilitated psycho-education sessions for coping with barriers and self-care, and provided resources to support patients' to organise their medication regimens. In addition, the Practice Nurses coordinated a multi-disciplinary team of physical and mental healthcare providers to provide tailored medical and mental healthcare. Growth curve analyses were used to assess outcomes, demonstrating significant improvements 12-months postintervention for the HR-QOL mental health subscale and viral load. However, non-significant improvements were present for the HR-QOL physical health subscale and immune functioning.

Van Der Meulen et al. [87] offered six bimonthly 45-min nurse-led, problem-focused counselling sessions for depressive symptoms to HNC patients $(n=205)$ over a 12-month period. Three experienced oncology nurses received a one-day training course from two psychologists and one investigator on the 'Nurse Counselling and After Intervention'. Session recordings were reviewed every 2 months to assess intervention quality. The intervention focussed on managing the physical, psychological and social consequences of $\mathrm{HNC}$, restructuring illness cognitions and beliefs, education and behavioural relaxation training, and providing emotional support. Significant improvements were present immediately post-intervention (both in the overall sample and depressive subgroup) for the primary endpoint of depressive symptoms and secondary endpoint of overall physical symptoms.

\section{Discussion}

\section{General statement}

The review aimed to examine the effectiveness of psychological interventions to improve specific or generic components of QOL, HR-QOL and/or MWb in people with LTC, with a view to advising LTC healthcare provision. The findings, strengths, limitations and implications of studies, and the strengths and limitations of the current review and rapid SR procedure, are discussed.

\section{Short-term interventions (0-3 months) Six-week self-regulation for older adult asthmatics}

Baptist et al. [82] trained health educators on a two-day programme which enabled them to facilitate a six-week self-regulation intervention. As a consequence of the selfregulation intervention, significant improvements occurred for older adults' overall asthma-related QOL and control up to 12-months post-intervention. The key hallmarks of the self-regulation approach was to facilitate patients' self-identification of a specific condition-related issue and potential barriers and goals, in order to provide tailored support and increase patients' self-efficacy over their condition. This approach has also been used to achieve positive outcomes for heart disease and medical noncompliance in older adults [90,91]. Therefore, when combined with the low attrition rate (7\%) [82] and selfregulation concepts not being unique to asthma [92], selfregulation provides promise as an effective and acceptable form of intervention to improve QOL in older adults. Despite receiving 'Strong' ratings for all but one quality component, the study received a 'Weak' 'Selection Bias' rating due to only $54 \%$ of those approached agreeing to participate, which may have two potential implications. First, this may indicate a lack of interest in self-regulation interventions potentially due to this approach differing from anticipated traditional asthma care approaches [82]. Second, while double-blinding improves methodological quality [93], a lack of awareness of intervention procedures and potential benefits may have impact enrolment. Additionally, as highlighted by the authors, the study was limited by using a single site and required a certain threshold of patient communicative ability to contribute to 
group discussions. Therefore, while additional studies and a cost-benefit analysis would be required to determine the efficacy of larger scale programmes, and consideration is required for the enrolment confounds, the study demonstrated that a short-term, health educator-led selfregulation intervention may have promising implications for LTC healthcare.

\section{Six-week structured self-management for CHF}

Smeulders et al.'s [85] 6-week structured self-management intervention, co-facilitated by a trained cardiac nurse specialist and a CHF peer role model, significantly improved cardiac-specific QOL immediately post-intervention. However, effects were not maintained at 6- or 12-months follow-up, and no other QOL improvements occurred. Despite having four 'Strong' components, the study received an overall 'Weak' EPHPP quality rating due to unspecified 'Blinding' of patients and clinicians, and a 'Selection Bias' as only $44 \%$ of eligible patients participated. As justification for non-participation varied considerably - from a lack of interest to physical, psychosocial or cognitive problems preventing participation - a qualitative study to further explore enrolment issues may be beneficial to determine whether the intervention was sufficiently tailored to complex CHF needs. While the authors proposed that non-significant effects may have resulted from insufficient intervention length or intensity above the "relatively high level" of Dutch standard care, a similar medium-term (15 weeks) self-management intervention improved physical but not emotional QOL [94]. Therefore, despite positive short-term results, further research is required to understand the mechanisms behind the low participation and lack of long-term QOL effects for structured self-management, with a view to using this to develop and trial more tailored interventions.

\section{Overall short-term interventions}

Despite both short-term interventions reviewed [82, 85] comprising 6-week programmes, considerable differences were present between-interventions that may have influenced outcomes. First, the self-regulation intervention was solely facilitated by health educators, while the CHF intervention was co-facilitated by a nurse and a patient 'peer leader'. While peer leaders were trained to effectively facilitate the intervention, potential differences in pre-existing knowledge and experience associated with not being a trained healthcare professional may have influenced the content, approach and style of programme adopted, and subsequently QOL outcomes. Second, research into the mechanisms behind why the 2-day (but not the 4-day) training resulted in significant long-term QOL improvement would be beneficial. Three possible explanations for this include potential differences in the quality of training, that health educators may benefit more from short-term training than nurses and/or peer leaders, and/or that additional information provided during the longer training may have resulted in a more structured but less tailored approach being adopted with patients. Third, as asthma and CHF differ considerably in emotional, physical and social outcomes [95, 96], this may have impacted the long-term maintenance of intervention effects post-intervention and consequently QOL outcomes. Fourth, methodological differences may have impacted outcomes due to the discrepancy between Blank et al.'s [82] 'Moderate' and Smuelders et al.'s [85] 'Weak' EPHPP quality ratings. However, despite considerable differences, both studies demonstrated that interventions which actively engage and involve the patient in their care may significantly improve at least short-term QOL, and that, while achieving initial buy-in for these types of interventions may be challenging, once enrolled attrition rates were low. Therefore, while cost-benefit analyses and further research are required to determine viability and overcome current limitations, short-term psychological interventions that actively involve patients demonstrated initial promise for improving QOL, with self-regulation demonstrating particular promise.

\section{Medium-term interventions (3-12 months) \\ Three-month CBT for medically unexplained symptoms}

Escobar et al.'s [84] structured CBT therapist-led intervention for MUPS significantly improved patient-rated depression and somatic symptoms, and clinician-rated severity of symptoms, immediately post-intervention. However, only improvements to patient-rated somatic symptoms were maintained 6-months post-intervention. While depressive and somatic symptom improvements were anticipated as CBT is widely advocated for depression, the improvements in both patient- and clinicianrated MUPS symptoms potentially indicate additional benefits for short-term perceived behavioural and cognitive control. Despite positive results, achieving patient buy-in was problematic as only $41 \%$ of eligible patients enrolled with an attrition rate of $45 \%$. While the justification for this was not discussed, the study proposed that future programmes may benefit from using a staged-approach to tailor the intervention to patients' needs, use of other services, costs, and the delivery setting. As MUPS patients do not benefit from reassurance alone [97] and a similar 6-week CBT programme for Breast Cancer patients demonstrated non-significant results [98], this highlights the need for at least moderate-length, tailored CBT-based interventions that are tailored to patients' needs. Therefore, while research is required to overcome the confounds of participation and long-term effect maintenance, and to determine how to feasibly implement the complex and time- 
consuming intervention in practice, $\mathrm{CBT}$ demonstrated promise for improving QOL in LTC.

\section{Six-month BWM/PCST for knee osteoarthritis}

Somers et al.'s [86] clinical psychologist-led 24-week combined PCST and BWM intervention demonstrated significant improvements 12-months post-intervention for the QOL components of arthritis- and weightspecific self-efficacy, pain symptoms and catastrophizing, physical disability and stiffness, weight, and BMI compared to UCC. Additionally, the combined intervention was significantly more effective than the individual interventions for the aforementioned outcomes; excluding PCST for pain catastrophizing and one pain measure. This demonstrates that by conducting a programme which not only targets LTCs' physical components, but also enables people to cope with the psychological effects and consequences, significantly improves both physical and psychological QOL. However, despite being one of only two studies reviewed to receive a 'Strong' quality rating, the study was confounded by the combined condition receiving double the intervention dosage than individual conditions. Additionally, as interventions were facilitated by highly trained clinical psychologists, additional research and a cost-benefit analysis comparing this approach with training existing staff involved in arthritis healthcare to provide the intervention would be beneficial. Therefore, while research for potential dose and expertise effects is required, the study demonstrated efficacy for a medium-term intervention to improve QOL 12-months post-intervention through targeting both the physical and psychological components of LTC.

\section{Overall medium-term interventions}

Overall, the medium-term studies [84, 86] demonstrated effectiveness for interventions delivered by psychologically trained staff to improve QOL in LTC, with CBT resulting in short-term improvements and a combined physical and psychological intervention resulting in improvements 12-months post-intervention. While these studies highlighted the need for medium-term psychological interventions to be tailored to LTC patients' physical and psychological needs in order to actively involve patients in their healthcare, three considerations are required. First, differences were present in the quality of studies, with Escobar et al. [84] receiving a 'Moderate' quality rating and Somers et al. [86] a 'Strong' rating. As this stemmed purely from the CBT-therapist intervention experiencing more problematic 'Withdrawals $\mathcal{E}$ Dropouts' [84], future research into the mechanisms behind this difference would be beneficial. Second, despite both LTC having profound physical and psychological consequences, current understanding of the causes and consequences of MUPS is less well defined than for knee osteoarthritis, which may have impacted outcomes [84, 86]. Third, while the positive outcomes provide an important foundation for research to build upon, consideration is required for the level of staff input and training required to conduct such programmes. As becoming a chartered psychologist or CBT therapist typically takes at least 6-7 years of study and training in addition to vocational work, both programmes required highly specialised staff. While this appears beneficial for QOL outcomes, this raises potential practicality issues for healthcare implementation as considerations would be required to determine capacity, practicality and financial viability within existing or additional services. However, as Somers et al. [86] demonstrated greater improvements based on psychological intervention dosage, this highlights a potential opportunity to utilise psychological principles to improve QOL outcomes for LTC. Therefore, careful consideration is required for the implementation of medium-term interventions using psychologically trained staff; however, the positive effects for both physical and psychological QOL indicate promise for healthcare.

\section{Long-term interventions ( $\geq 12$ months) Twelve-month psycho-education and management for HIV} Blank et al.'s [83] 12-month, nurse-led community-based psycho-education and healthcare management intervention for HIV patients demonstrated significant improvements for mental health QOL and immune functioning 12-months post-intervention. However, no effect was present for physical health QOL or viral load. The rationale behind the study was that reforms to healthcare provide a challenge but also an opportunity to redesign systems in a more integrated manner. Through training nurses to facilitate psycho-education while providing tailored access to relevant professions within a multidisciplinary healthcare team, significant improvements were present for condition-related immune functioning and mental health. However, future healthcare research would benefit from factoring in key confounds. First, as university-based nurses facilitated the intervention the additional research experience associated with this work setting may have influenced outcomes. Second, as viral load changes only occurred 12-months postintervention, consideration of optimal intervention and assessment duration is required. Finally, while assessing different constructs at different time points may be the most feasible approach within multi-disciplinary interventions, careful consideration is required for the effect this may have on analyses and attrition, as $75 \%$ of patients completed the QOL measure 12-months postintervention compared with only $61 \%$ providing biomarkers data. Therefore, while future work may benefit from overcoming practical confounds, altering existing 
services to provide psycho-education and tailored management of a multi-disciplinary team by nurses may be a feasible, cost-effective approach.

\section{Twelve-month counselling for HNC}

Van Der Meulen et al.'s [87] 12-month, nurse-led problem-focussed counselling programme significantly improved depressive and physical symptoms in HNC patients immediately post-intervention, with effects being more pronounced in the depressive-subgroup. As the authors proposed that those with greatest physical impairments were more likely to experience depressive symptoms and those with depressive symptoms benefited most from the intervention, problem-focussed counselling demonstrated efficacy both for the general sample and for those patients in greatest need. While the study was confounded by a 'Moderate' 'Selection Bias' with only $63 \%$ of eligible patients participating, it was one of only two studies to receive a 'Strong' overall rating and once enrolled attrition rates were low (13\%). Therefore, as low attrition supports the authors' claim that utilising nurse facilitators may not only reduce healthcare costs but also stigma, the intervention was feasible and cost-effective. Hence, due to the positive intervention effects a combined with the psychological elements of the interventions not being specific to $\mathrm{HNC}$, theory-based long-term nurse-facilitated interventions provide promise for LTC healthcare delivery.

\section{Overall long-term interventions}

Overall, the long-term studies [83, 87] demonstrated efficacy for long-term nurse-led interventions to improve QOL in LTC, with HNC counselling having significant post-intervention effects, and HIV psychoeducation and care management improving QOL 12months post-intervention. Despite differences in the format, content and delivery of interventions, significant QOL improvements were achieved through supporting nurses to facilitate interventions that enabled patients to develop the skills, knowledge and efficacy required to manage the physical and psychological components and consequences of their LTC. Furthermore, as both HIV and HNC are complex LTC that may have profound physical and mental effects and therefore require a large amount of medical support, the positive intervention effects provide promise for other complex LTC. As proposed by Van Der Meulen et al. [87], utilising nurses to provide long-term interventions may be both a financially and practically viable approach to implementing long-term psychological interventions, and may reduce stigma due to nurses already being intrinsically involved in LTC healthcare provision. However, consideration is require for the differences between Blank et al.'s [83] 'Moderate' and Van Der Meulen et al.'s [87] 'Strong' quality ratings, with this stemming from the 'Weak' and 'Strong' 'Withdrawals \& Dropouts' quality ratings respectively. Therefore, future research is required into the mechanisms behind between-study differences in enrolment and attrition despite both interventions utilizing nurse facilitators. Hence, long-term, nurse-led interventions which actively involve patients in their care and target both the physical and psychological constructs of LTC provide promise for healthcare. However, further research is required to determine the optimal approach to adopt in order to enhance patient enrolment for such programmes.

\section{General discussion Implications of Findings}

The studies reviewed demonstrated that psychological interventions for LTC varied considerably in terms of duration, population, methods, quality ratings, facilitators and long-term effectiveness. Descriptive analysis of findings indicated that all interventions resulted in significant improvements to at least one component of QOL immediately-post intervention. Furthermore, the 6-week health educator self-regulation intervention for asthma [82], 6-month clinical psychologist-led combined PCST-BWM intervention for knee osteoarthritis [86], and 12-month nurse-led psycho-education and care management intervention for HIV [83] significantly improved QOL 12-months post-intervention. While further research is required to assess the mechanisms behind differences in the effectiveness of interventions and the feasibility of implementing interventions in LTC healthcare, the findings indicate that psychological interventions utilising different formats, durations and facilitators which actively involve and enable patients to have self-efficacy over their care may result in significant QOL improvements for LTC patients.

In addition to the effectiveness of interventions, the studies have important implications for future research and healthcare. First, across studies enrolment in psychological interventions was low, with one study only successfully enrolling $41 \%$ of potential patients [84]. While blinding was often used to increase methodological quality, this may have influenced participation rates through blinding patients to the potential components, goals and benefits of interventions. Additionally, at present LTC treatments typically promote pharmacological and/or medical treatments, with psychological interventions promoted as secondarily $[1-3,5-7,11,19$, $22,23,26]$. This may promote patients to seek quick-fix treatments and requires a change in approach in order to enhance participation in psychological interventions. Further, as only 6 RCTs from 2006 to February2016 were deemed suitable based on the inclusion/exclusion criteria, coupled with the review demonstrating that psychological 
interventions may improve QOL across LTC, this review highlights the need for high-quality research into this area and the application of methods in healthcare. Hence, future research and interventions across LTC that attempt to build upon the positive findings and resolve methodological confounds is recommended in order to build a greater evidence base for the effectiveness of psychological interventions on LTC.

\section{General Strengths and Limitations}

Many of the strengths of the review may also be regarded as limitations. First, an ante hoc decision was made to include only RCTs with a UCC in order to ensure that only high methodological quality studies were included and valid comparisons could be made between interventions despite considerable differences in the LTC targeted [67]. Furthermore, in order to ensure that only the most up-to-date research was assessed, only studies spanning the previous 10 years (2006 to February 2016) were included. While discussions were conducted with relevant experts (within Public Health, Health Psychology and Publishing) prior to the review to set a strict inclusion/exclusion criterion for only the most relevant research, it is possible that important and interesting studies, findings and interventions may have been excluded. Additionally, in order to improve the reliability of findings, only studies that directly targeted LTC patients for both the intervention and assessment were included. However, this may also have reduced the number of interventions through excluding those that indirectly target or assess patients through clinicians, carers or family members, such as communicative or learning disorder populations who may benefit from psychological interventions but are unable to communicate effects. Finally, while he COCHRANE data extraction framework is well validated and used across disciplines [73], the EPHPP quality assessment tool was used as the review aimed to guide public health policy [74]. However, as the review assessed psychological interventions, alternative tools may potentially have been more appropriate and may have resulted in different quality ratings. For example, Smeulders et al. [85] received a 'Weak' rating despite demonstrating four 'Strong' components, and Van Der Meulen et al. [87] received a 'Strong' rating despite only stating significance values as ' $p \leq 0.05$ '. Therefore, future replications and expansions should attempt to build upon the strengths, and generate solutions for the limitations, of the review in order to improve upon the quality of the review.

\section{Rapid systematic review strengths and limitations}

Previous research has discussed the relative strengths and limitations of the rapid SR approach compared to traditional SRs [70-72]. One primary benefit of this methodology is that it may be used to assess research and formulate conclusions that influence healthcare policy within a time-frame and budget that would not be possible using traditional methods. While significant work was subsequently conducted to improve the review to publication standard, this methodology allowed the review to progress from defining potential search parameters to providing a first draft to healthcare stakeholders within three-months. Rapid SRs may potentially suffer from using a non-iterative search strategy, narrow timeframe for retrieval, not performing quality analysis, and limiting consultation with experts. However, the present review did not suffer from these confounds as a strict ante hoc criteria was set and adhered to, and various contacts (Public Health, Health Psychology etc.) were sought out to discuss the suitability of the review. Therefore, active efforts were made to strengthen methodology by ensuring that many potential confounds of rapid SRs were accounted for.

Despite attempts to maintain as high quality methodology as possible, implicit limitations are associated with one researcher being involved until data extraction. First, practical constraints meant that grey literature, reference lists and additional databases were not searched, which may have provided additional findings. Furthermore, while all possible effort were made to maintain accuracy, 'human error' and 'selection bias' are possible, and as only articles published in English were included 'publication' and 'language' biases are also possible. However, given the relative strengths and weaknesses of rapid SRs, and that the review was completed during NHS employment (See Authors' Information), overall utilising rapid SR methodology was useful for an initial study. Therefore, future attempts should be made to replicate and expand upon the findings using a larger research team to limit the aforementioned confounds through continuing to utilise a strict ante hoc criteria.

\section{Conclusions}

The studies reviewed demonstrated promising results for utilising psychological interventions to improve QOL in LTC patients, with short-, medium- and long-term interventions that promote patient involvement demonstrating positive outcomes. While confounds were present which require resolution, particularly with low participation from eligible patients, the positive results indicated that with high-quality methodology, actively involving patients in their care and tailoring of interventions to patients' needs, psychological interventions may improve QOL in LTC. Hence, future studies should assess the efficacy of tailored interventions utilising different formats, durations, and facilitators to improve QOL in LTC, while the development and promotion of services should be promoted to utilise psychological interventions to supplement medical care, 


\section{Abbreviations}

BWM: Behavioural Weight Management.; CBT: Cognitive Behavioural Therapy; CHF: Congestive Heart Failure; EPHPP: Effective Public Health Practice Project; HIV: Human Immunodeficiency Virus; HNC: Head \& Neck Cancer; HRQOL: Health-Related Quality of Life; LTC: Long-Term Conditions; MUPS: Medically Unexplained Physical Symptoms; MWB: Mental Wellbeing; PCST: Pain Coping Skills Training; QOL: Quality of Life; RCT: Randomised Controlled Trial; SR: Systematic Review; UCC: Usual Care Control

\section{Acknowledgements}

NA would like to thank Julie Murray and Dr. Allyson McCollam at NHS Borders, and Dr. Hannah Dale and Dr. Lloyd Wallace at NHS Education for Scotland, for their ongoing support and advice throughout the review.

\section{Funding}

The authors declare that they did not receive any financial support for the present study.

\section{Availability of Data and Materials}

All data generated or analysed during this study are included in this published article.

\section{Authors' Contributions}

NA was involved in all processes involved in the rapid SR, including: design, research, development, search, extraction, collation, analyses and reporting formulation. GO provided supervision, advice, feedback, and contributions towards all processes from data extraction onwards. Both authors read and approved the final manuscript.

\section{Authors' Information}

NA graduated with a BSc (Hons.) Psychology degree from the University of Dundee in 2014, before graduating with a MSc Health Psychology degree from the University of St Andrews in 2015. NA conducted the review as part of employment as a Trainee Health Psychologist in NHS Borders, with research being conducted in affiliation with the University of St Andrews. NA aims to achieve Chartership as a British Psychological Society and Health \& Care Professionals Council registered Health Psychologist in June 2018. $\mathrm{GO}$ is a Chartered Health Psychologist and is a Senior Lecturer in Health Psychology within the University of St Andrews School of Medicine Division of Population and Behavioural Health Sciences.

\section{Ethics Approval and Consent to Participate}

Not applicable.

\section{Consent for Publication}

Not applicable.

\section{Competing Interests}

The authors declare that they have no competing interests.

\section{Publisher's Note}

Springer Nature remains neutral with regard to jurisdictional claims in published maps and institutional affiliations.

\section{Received: 4 October 2017 Accepted: 13 March 2018}

Published online: 27 March 2018

\section{References}

1. Burns H. Improving the health and wellbeing of people with long term conditions: A national action plan. Long Term Conditions Action plan. Scottish Government; 2009. http://www.gov.scot/Resource/Doc/294270/ 0090939.pdf. Accessed 17 Oct 2016

2. Timmerick TC. Dictionary of health services management. 2nd ed. Owings Mills: Maryland; 1987.

3. Gray L, Leyland A. Volume 1 main report. In: Scottish Health Survey 2012 Scottish Government. 2013. http://www.gov.scot/Resource/0043/00434590. pdf. Accessed 10.02.2016

4. Janssen F. Cohort patterns in mortality trends among elderly in seven European countries, 1950-99. Int J Epidemiol. 2005;34(5):1149-59.

5. Kinsella KG, Phillips DR. Global aging: The challenge of success. 1st ed. Population Reference Bureau: Washington; 2005.
6. Nolte E, McKee M. Caring for people with chronic conditions: A health system perspective. $1^{\text {st }}$ ed. London: McGraw-Hill. Education. 2008;

7. Goodwin N, Curry N, Naylor C, Ross S, Duldig W. Managing people with long-term conditions. In: An inquiry into quality of general practice in England. The Kings Fund. 2010. http://www.kingsfund.org.uk/sites/files/kf/ field/field_document/managing-people-long-term-conditions-gp-inquiryresearch-paper-mar11.pdf. Accessed 17 Oct 2016.

8. Hackett ML, Yapa C, Parag V, Anderson CS. Frequency of depression after stroke. Stroke. 2005;36:1330-40.

9. Haines L, Wan KC, Lynn R, Barrett TG, Shield JP. Rising incidence of type 2 diabetes in children in the UK. Diabetes Care. 2007:30:1097-101.

10. Wolfe CD. The impact of stroke. Br Med Bull. 2000;56(2):275-86.

11. Choice Access Team. Understanding the benefits. In: Improving access to psychological therapies (IAPT) commissioning toolkit. Department of Health. 2008. https://www.uea.ac.uk/documents/246046/11991919/IAPT +Commissioning+Toolkit+2008+.pdf/cc6a4f24-dc6b-45d9-a631-ffdd075c6f0a. Accessed 17 Oct 2016

12. Halpin DM, Miravitlles M. Chronic obstructive pulmonary disease: The disease and its burden to society. Prom Am Tharacic Soc. 2006:3(7):619-23.

13. Wimo A, Winblad B, Aguero-Torres H, Von Strauss E. The magnitude of dementia occurrence in the world. 2003. Alzheimers Dis Assoc Disord. 2003;17(2):63-7.

14. Akehurst RL, Brazier JE, Mathers N, O'Keefe C, Kaltenthaler E, Morgan A, et al. Health-related quality of life and cost impact of irritable bowel syndrome in a UK primary care setting. PharmacoEconomics. 2002;20(7):455-62.

15. Nimnuan C, Hotopf M, Wessely S. Medically unexplained symptoms: An epidemiological study in seven specialities. J Psychosom Res. 2001;51(1):361-7.

16. Konnopka A, Schaefert R, Heinrich S, Kaugmann C, Luppa M, Herzog W, et al. Economics of medically unexplained symptoms: A systematic review of the literature. Psychother Psychosom. 2012:81(5):265-75.

17. Feinstein AR. The pre-therapeutic classification of co-morbidity in chronic disease. J Chronic Dis. 1970;23(7):455-68.

18. Fortin M, Bravo G, Hudon C, Vanasse A, Lapoint L. Prevalence of multi-morbidity among adults seen in family practice. Ann Fam Med. 2005:3(3):223-8.

19. Boerma JT, Stansfield SK. Health statistics now: are we making the right investments? Lancet. 2007:369(9563):779-86.

20. Murray CJ. Towards good practice for health statistics: lessons from the Millennium Development Goal health indicators. Lancet. 2007:369(9564):862-73.

21. Alonso J, Ferrer M, Gandbeck B, Ware Jr JE, Aaronson NK, Mosconi P, et al. Health-related quality of life associated with chronic conditions in eight countries: results from the International Quality of Life Assessment (IQOLA) Project. Qual Life Res. 2004;13(2):282-98.

22. Ogden J. Health Psychology: A textbook. 5th ed. London: McGraw-Hill Education (UK; 2012.

23. Albrecht GL, Fitzpatrick R. A sociological perspective on health-related quality of life research. In: Advances in medical sociology, quality of life in health care London: Jai Press; 1994. p. 1-21.

24. Bradley C. Importance of differentiating health status from quality of life Lancet. 2001:357(9249):7-8.

25. WHOQoL Group. The development of the World Health Organisation quality of life assessment instrument (the WHOQOL). In: Quality of life assessment: International Perspectives. Berlin: Springer Berlin Heidelberg; 1994. p. 41-57.

26. Aaronson NK, Ahmedzai S, Bergman B, Bullinger M, Cull A, Duez NJ, et al. The European Organisation for Research and Treatment of Cancer QLQ-C30: A quality of life instrument for use in international clinical trials in oncology. J Natl Cancer Inst. 1993;85(5):365-76.

27. Fallowfield $L$. The quality of life: The missing measurement in health care. 1st ed. London: Souvenir Press; 1990.

28. Goldberg D, Williams P. General health questionnaire (GHQ). In: Swindon: nferNelson; 1988.

29. Hays RD. RAND-36 health status inventory. San Antonio: Psychological Corporation; 1998

30. Hickey AM, Bury G, O'Boyle CA, Bradley F, O'Kelly FD, Shannon W. A new short form individual quality of life measure (SEIQoL-DW): Application in a cohort of individuals with HIV/AIDS. BMJ. 1996;313(7048):29.

31. Skevington SM, O'Connell KA. Measuring quality of life in HIV and AIDS: A review of the recent literature. Psychol Health. 2003;18(3):331-50.

32. Skevington SM, O'Connell KA. Can we identify the poorest quality of life? Assessing the importance of quality of life using the WHOOOL-100. Qual Life Res. 2004;13(1):23-34. 
33. Zigmond AS, Snaith RP. The hospital anxiety and depression scale. Acta Psychiatr Scand. 1983;67(6):361-70.

34. Furlong WJ, Feeny DH, Torrance GW, Barr RD. The Health Utilities Index $\left(\mathrm{HUI}{ }^{\oplus}\right)$ system for assessing health-related quality of life in clinical studies. Ann Med. 2001;33(5):375-84.

35. Kaplan RM, Anderson JP, Ganiats TG. The quality of well-being scale: rationale for a single quality of life index. In: Quality of life assessment: Key issues in the 1990s. Amsterdam: Springer; 1993. p. 65-94.

36. Ware Jr JE, Kosinski M, Keller SD. A 12-item short-form health survey: Construction of scales and preliminary tests of reliability and validity. Med Care. 1996;34(3):220-33.

37. American Psychiatric Association. Diagnostic and Statistical Manual, $5^{\text {th }}$ Edition (DSM-V). 5th ed. Washington: American Psychiatric Association; 2013.

38. Bener A, Al-Kazaz M, Ftouni D, Al-Harthy M, Dafeeah EE. Diagnostic overlaps of depressive, anxiety, stress and somatoform disorders in primary care. Asia Pac Psychiatry. 2013;5(1):E29-8.

39. Gilbert P. Depression: The evolution of powerlessness. 1st ed. Hove: Psychological Press; 2014.

40. Spielberger CD. Anxiety: Current trends in theory and research. 1st ed. Philadelphia: Elsevier; 2013.

41. Jackson-Koku G. Beck Depression Inventory. Occup Med. 2016;66(2):174-5.

42. Steer RA, Beck AT. Beck Anxiety Inventory. In: Zalaquett CP, Wood RJ, editors. Evaluating stress: A book of resources. Lanham: Scarecrow Education; 1997. p. 23-40.

43. Tennant R, Hiller L, Fishwick R, Platt S, Jospeh S, Weich S, et al. The WarwickEdinburgh mental well-being scale (WEMWBS): development and UK validation. Health Qual Life Outcomes. 2007;5(1):1.

44. Moussavi S, Chatterji S, Verdes E, Tandon A, Patel V, Ustun B. Depression, chronic diseases, and decrements in health: Results from the World Health Surveys. Lancet. 2007:370(9590):851-8.

45. Sareen J, Jabobi F, Cox BJ, Belik SL, Clara I, Stein MB. Disability and poor quality of life associated with comorbid anxiety disorders and physical conditions. Arch Intern Med. 2006;166(19):2109-16.

46. Ganz PA, Desmond KA, Leedham B, Rowland JH, Meyerowtiz BE, Belin TR. Quality of life in long-term, disease-free survivors of breast cancer: A followup study. J Natl Cancer Inst. 2002;94(1):39-49.

47. Karakoyun-Celik O, Gorken I, Sahin S, Orcin E, Alanyali H, Kinay M. Depression and anxiety levels in woman under follow-up for breast cancer: a relationship to coping with cancer and quality of life. Med Oncol. 2010;27(1):108-13.

48. Margolis G, Goodman RL, Rubin A. Psychological effects on breast-conserving cancer treatment and mastectomy. Psychosomatic. 1990;31(1):33-9.

49. Spiegel D, Giese-Davis J. Depression and cancer: mechanisms and disease progression. Biol Psychiatry. 2003;54(3):269-82.

50. Watson M, Haviland JS, Greer S, Davidson J, Bliss JM. Influence of psychological response on survival in breast cancer: a population-based cohort study. Lancet. 1999;354(9187):1331-6.

51. Davies SJ, Jackson PR, Potokar J, Nutt DJ. Treatment of anxiety and depressive disorders in patients with cardiovascular disease. BMJ. 2004; 328(7445):939-43.

52. Frasure-Smith N, Lesperance F, Juneau M, Talajic M, Bourassa MG. Gender, depression, and one-year prognosis after myocardial infarction. Psychosom Med. 1999;61(1):26-37.

53. Burns H. Health in Scotland 2007: Annual report of the chief medical officer. 1st ed. The Scottish Government: Edinburgh; 2009.

54. Sanders C, Egger M, Donovan J, Tallon D, Frankel S. Reporting on quality of life in randomised controlled trials: Bibliographic study. BMJ. 1998:317(7167):1191-4.

55. World Health Organisation. 2008-2013 action plan for the global strategy for the prevention and control of non-communicable disease: Prevent and control cardiovascular diseases, cancers, chronic respiratory diseases and diabetes. In: 2008-2013 Action Plan. World Health Organisation. 2009. http://apps.who.int/iris/bitstream/10665/44009/1/9789241597418_eng.pdf. Accessed 18 July 2016. Accessed 17 Oct 2016.

56. Cohen S, Herbert TB. Health Psychology: Psychological factors and physical disease from the perspective of human psychoneuroimmunology. Annu Rev Psychol. 1996:47(1):113-42

57. Prince M, Patel V, Saxena S, Maj M, Maselko J, Phillips MR, et al. No health without mental health. Lancet. 2007;370(9590):859-77.

58. Galway K, Black A, Cantwell M, Cardwell CR, Mills M, Donnelly M. Psychosocial interventions to improve quality of life and emotional wellbeing for recently diagnosed cancer patients. Psycho-Oncology. 2013;22:253-4.
59. Happell B, Davies C, Scott D. Health behaviour interventions to improve physical health in individuals diagnosed with mental illness: A systematic review. Int J Ment Health Nurs. 2012;21(3):236-47.

60. Harkness E, Macdonald W, Valderas J, Coventry P, Gask L, Bower P. Identifying psychosocial interventions that improve both physical and mental health in patients with diabetes a systematic review and metaanalysis. Diabetes Care. 2010;33(4):926-30.

61. Hutchison AJ, Breckon JD. A review of telephone coaching services for people with long-term conditions. J Telemed Telecar. 2011;17(8):451-8.

62. Rehse B, Pukrop R. Effects of psychosocial interventions on quality of life in adult cancer patients: meta analysis of 37 published controlled outcome studies. Patient Educ Couns. 2003;50(2):179-86.

63. Simpson R, Booth J, Lawrence M, Byrne S, Mair F, Mercer S. Mindfulness based interventions in multiple sclerosis-a systematic review. BMC Neurol. 2014;14(1):165.

64. Stinson J, Wilson R, Gill N, Yamada J, Holt J. A systematic review of internetbased self-management interventions for youth with health conditions. J Pediatr Psychol. 2009;34(5):495-510.

65. Trautmann E, Lackschewitz H, Kröner-Herwig B. Psychological treatment of recurrent headache in children and adolescents-a meta-analysis. Cephalalgia. 2006;26(12):1411-26.

66. Van Beugen S, Ferwerda M, Hoeve D, Rovers MM, Spillekom-Van Koulil S, Van Middendorp $\mathrm{H}$, et al. Internet-based cognitive behavioral therapy for patients with chronic somatic conditions: a meta-analytic review. J Med Internet Res. 2014;16(3):e88.

67. Sibbald B, Roland M. Understanding controlled trials. Why are randomised controlled trials important? BMJ. 1998;316(7126):201.

68. Hoffman BM, Papas RK, Charkoff DK, Kerns RD. Meta-analysis of psychological interventions for chronic low back pain. Health Psychol. 2007;26(1):1.

69. Rose MJ, Reilly JP, Pennie B, Bowen-Jones K, Stanley IM, Slade PD. Chronic low back pain rehabilitation programs: a study of the optimum duration of treatment and a comparison of group and individual therapy. Spine J. 1997; 22(19):2246-51.

70. Ganaan R, Ciliska D, Thomas H. Expediting systematic reviews: Methods and implications of rapid reviews. Implement Sci. 2010;5(1):56.

71. Khangura S, Konnyu K, Cushman R, Grimshaw J, Mosher D. Evidence summaries: The evolution of a rapid review approach. Syst Rev. 2012:1(1):1.

72. Featherstone RM, Dryden DM, Foisy M, Guise JM, Mitchell MD, Paynter RA, et al. Advancing knowledge of rapid reviews: An analysis of results, conclusions and recommendations from published review articles examining rapid reviews. Syst Rev. 2015;4(1):1.

73. Higgins JP, Green S. Cochrane handbook for systematic reviews of interventions. 5th ed. Chichester: Wiley-Blackwell; 2008.

74. Thomas BH, Ciliska D, Dobbins M, Micucci S. A process for systematically reviewing the literature: providing the research evidence for public health nursing interventions. Worldviews Evid Based Nurs. 2004;1(3);176-84.

75. Braeken AP, Kempen Gl, Eekers DB, Houben R, Gils GC, Ambergen T, et al. Psychosocial screening effects on health-related outcomes in patients receiving radiotherapy. A cluster randomised trial. Psychooncology. 2013; 22(12):2736-46.

76. Jensen AM, Ramasamy A, Hall MW. Improving General Flexibility with a Mind-Body Approach: A Randomized, Controlled Trial Using Neuro Emotional Technique ${ }^{\oplus}$. J Strength Cond Res. 2012;26(8):2103-12.

77. Lawler SP, Cameron LD. A randomized, controlled trial of massage therapy as a treatment for migraine. Ann Behav Med. 2006;32(1):50-9.

78. Mosher CE, Lipkus I, Sloane R, Snyder DC, Lobach DF, Demark-Wahnefried W. Long-term outcomes of the FRESH START trial: Exploring the role of selfefficacy in cancer survivors' maintenance of dietary practice and physical activity. Psychooncology. 2013;22(4):876-85.

79. Pulgaron ER, Salamon KS, Patterson CA, Barakat LP. A problem-solving intervention for children with persistent asthma: A pilot of a randomized trial at a pediatric summer camp. J Asthma. 2010;47(9):1031-9.

80. Tabolli S, Pagliarello C, Sampogna F, Di Pietro C, Abeni D, GISED ICS. Evaluation of the impact of writing exercises and education interventions on quality of life in patients with psoriasis. Value Health. 2011;14(7):A509-10.

81. Yardley L, Joseph J, Michie S, Weal M, Wills G, Little P. Evaluation of a Webbased intervention providing tailored advice for self-management of minor respiratory symptoms: exploratory randomized controlled trial. J Med Internet Res. 2010;12(4):e66.

82. Baptist A, Ross JA, Yany Y, Song PX, Clark NM. A randomized controlled trial of a self-regulation intervention for older adults with asthma. J Am Geriatr Soc. 2013;61(5):747-53. 
83. Blank MB, Hennessy M, Eisenberg MM. Increasing quality of life and reducing HIV burden: The PATH+ intervention. AIDS Behav. 2014;18(4):716-25.

84. Escobar Jl, Gara MA, Diaz-Martinez AM, Interian A, Warman M, Allen LA, et al. Effectiveness of a time-limited cognitive behaviour therapy-type intervention among primary care patients with medically unexplained symptoms. Ann Fam Med. 2007;5(4):328-35.

85. Smeulders ES, Van Haastregt J, Ambergen T, Uszko-Lencer NH, JanssenBoyce JJ, Gorgels AP, et al. Nurse-led self-management group programme for patients with congestive heart failure: randomized controlled trial. J Adv Nurs. 2010;66(7):1487-99.

86. Somers TJ, Blumenthal JA, Guilak F, Kraus VB, Schmitt DO, Babyak MA, et al. Pain coping skills training and lifestyle behavioral weight management in patients with knee osteoarthritis: a randomized controlled study. Pain. 2012; 153(6):1199-209

87. Van Der Meulen IC, May AM, Ros WJ, Oosterom M, Hordijk GJ, Koole R, et al. One-year effect of a nurse-led psychosocial intervention on depressive symptoms in patients with head and neck cancer: a randomized controlled trial. Oncologist. 2013;18(3):335-44.

88. Smeulders ES, Van Haastregt JC, Van Hoef EF, Van Eijk JT, Kempen Gl. Evaluation of a self-management programme for congestive heart failure patients: Design of a randomised controlled trial. BMC Health Serv Res. 2006;6(1):91.

89. Brown AJ, Smith LT, Craighead LW. Appetite awareness as a mediator in an eating disorders prevention programme. Eat Disord. 2010;18(4):285-301.

90. Janevic MR, Janz NK, Kaciroti N, Dodge JA, Keteyian SJ, Mosca L, et al. Exercise self-regulation among older women participating in a heart disease-management intervention. J Women Aging. 2010;22(4):255-72.

91. Kucukarslan SN, Thomas S, Bazzi A, Virant-Young D. Using self-regulation theory to examine patient goals, barriers and facilitators for taking medicine. Patient. 2009;2(4):211-20.

92. Sabaté E. Adherence to long-term therapies: Evidence for action. Geneva: World Health Organisation; 2003.

93. Boot WR, Simons DJ, Stothart C, Stutts C. The pervasive problem with placebos in psychology: Why active control groups are not sufficient to rule out placebo effects. Perspect Psychol Sci. 2013;8(4):445-54.

94. Shively M, Kodiath M, Smith TL, Kelly A, Bone P, Fettely L, et al. Effect of behavioural management on quality of life in mild heart failure. Patient Educ Couns. 2005;58(1):27-34.

95. Jones SC, Iverson D, Burns P, Evers U, Caputi P, Morgan S. Asthma ageing: An end user's perspective-the perception problems with the management of asthma in the elderly. Clin Exp Allergy. 2011;41(4):471-81.

96. Yu DS, Lee DT, Kwong AN, Thompson DR, Woo J. Living with chronic heart failure: a review of qualitative studies of older people. J Adv Nurs. 2008; 61(5):474-83.

97. Rief W, Heitmüller AM, Reisberg K, Rüddel H. Why reassurance fails in patients with explained symptoms-an experimental investigation of remembered probabilities. PLoS Med. 2006;3(8):e269.

98. McKiernan A, Steggles S, Guerin S, Carr A. A controlled trial of group cognitive behaviour therapy for Irish breast cancer patients. J Psychosoc Oncol. 2010;28(2):143-56.

\section{Submit your next manuscript to BioMed Central and we will help you at every step:}

- We accept pre-submission inquiries

- Our selector tool helps you to find the most relevant journal

- We provide round the clock customer support

- Convenient online submission

- Thorough peer review

- Inclusion in PubMed and all major indexing services

- Maximum visibility for your research

Submit your manuscript at www.biomedcentral.com/submit
Biomed Central 